\title{
The hard sphere view of the outer core
}

\author{
George Helffrich
}

\begin{abstract}
The hard sphere model for liquids attempts to capture the physical behavior of a real liquid in a simple conceptual model: a fluid of fixed size spheres that only interact repulsively when they come into contact. Is the model good enough to use for modeling internal planetary structure? To answer this question, I survey variants of hard sphere liquid theory by applying them to the Earth's outer core to determine which of them explains wavespeeds in the outer core best. The variants explored here are the Carnahan-Starling hard sphere model, the Mansoori-Canfield extension to hard sphere mixtures, the transition metal hard sphere liquid, and the Lennard-Jones hard sphere liquid with attractive forces. With an empirical addition of a temperature dependence to the liquid's hard sphere diameter, all of the variants explored can replicate wavespeeds in most of the radius range of the outer core. The hard sphere model for liquid transition metals explains the wavespeed best because it yields a mean liquid atomic weight of 48.8 $\mathrm{g} \mathrm{mol}^{-1}$ at $10 \mathrm{wt} \%$ light element abundance in the core which is in good cosmochemical agreement with core light element models. Other variants also fit core wavespeeds but require implausibly low liquid mean atomic weight implying excessive incorporation of hydrogen or helium in the core. Applied to the detailed wavespeed structure of the Earth's outermost outer core, the model suggests that the mean atomic weight could be reduced by up to $1.74 \%$ or the temperature could be increased by up to $400 \mathrm{~K}$ relative to an adiabatic profile, or there could be $8 \%$ fewer valence electrons in the liquid.
\end{abstract}

Keywords: Core; Seismology; Planetology; Hard sphere liquids; Liquid metal properties

\section{Background}

According to PREM (Dziewonski and Anderson 1981), the outer core appears to be essentially in a state of adiabatic self-compression, a state representing a convectively wellmixed and chemically homogeneously material. Birch (1952) established the link between finite strain theories of material behavior and the variation of wavespeed along an adiabat by deriving the dependence of bulk modulus on pressure, finding it related to the gravitational acceleration $(g)$ and the bulk sound speed derivative with depth $\left(d \mathrm{~V}_{\Phi} / d z\right)$.

In subsequent decades, physicists developed theories to describe fluids at high densities. They recognized that the dominant factors governing the behavior of high-density fluids were the repulsive interactions between the fluid constitutents (atoms or molecular species in the liquid) and the volume that those constitutents occupied in the liquid - the essential ingredients of the van der Waals equation of state for gases. A simple model captures these

\footnotetext{
Correspondence: george@elsi.jp

Earth-Life Science Institute, Tokyo Institute of Technology, 2-12-1 Ookayama, Meguro-ku, 152-8550 Tokyo, Japan
}

features: a fluid of spheres of fixed size that only interact repulsively when they come into contact (Carnahan and Starling 1969).

The Earth's core is not far from this idealization. It is a liquid metal comprised of iron alloyed with perhaps $10 \%$ by weight of a combination of light elements (Birch 1952, 1964). Liquid metals are characterized by a bulk fluid of positively charged ionic cores separated from their valence electrons that serve to neutralize the cores' charges (Hansen and McDonald 2013). Except for the valence electrons, this picture corresponds fairly well to a hard sphere fluid. For example, Stevenson (1980) used hard sphere theory to derive some fairly general properties of the core's density and wavespeed dependence on depth, and Helffrich (2014) used it to estimate diffusion coefficients in the core.

One motivation for using the hard sphere model is to estimate seismic wavespeeds in planets whose interior structure is only roughly known. The basic information from planetary discovery missions such as MESSENGER (Smith et al. 2012) typically consists of a mean density

\section{是 Springer}

(c) 2015 Helffrich; licensee Springer. This is an Open Access article distributed under the terms of the Creative Commons Attribution License (http://creativecommons.org/licenses/by/4.0), which permits unrestricted use, distribution, and reproduction in any medium, provided the original work is properly credited. 
and rotational moment of inertia. From this, a radial density model may be obtained (Smith et al. 2012). The radial density model then allows a hard sphere model to be applied to the liquid portions of the planet to predict seismic wavespeeds. In this way, preliminary seismic velocity models may be made to guide future planetary exploration missions that involve landers whose sensors include seismometers to determine the planet's detailed internal structure (Dehant et al. 2012).

Another motivation for this work is to have a means to study the wavespeed profile in the Earth's outer core directly. Because we know the aggregate elastic properties of the core quite well through decades of observational effort, it provides a good discriminator between successful and unsuccessful hard sphere theories. It is not obvious to the writer that a theory this simple should work at all. If one is suitable, it can serve as a reference for the core's constitution from which changes from an adiabatic, well-mixed state may be assessed. Wavespeeds lower than PREM observed at the top and bottom of the outer core (Helffrich and Kaneshima 2010; Ohtaki et al. 2012; Zou et al. 2008) are not expected from traditional views of core behavior (where lower wavespeeds are associated with higher densities and vice versa), though here I focus only the top of the core in order to prove the viability of the approach.

\section{Methods}

Data

I use the PREM model (Dziewonski and Anderson 1981) for values of density $(\rho)$, bulk sound speed $\left(\mathrm{V}_{\Phi}\right)$, and gravity $(g)$ through the outer core. As a reference model for the outermost outer core, Tanaka (2007) found that the PREM travel time predictions for SmKS (the family of arrivals that travel through the outer core and reflect $m-1$ times from the underside of the core-mantle boundary (CMB)) are the best among more recent radial velocity models, thus recommending its use. Gravity is not directly parameterized by PREM but may be fit with a polynomial form to radius when integrated from the density profile. The explicit expression for gravity $\left(\mathrm{m} \mathrm{s}^{-2}\right)$ in the outer core is

$$
\begin{aligned}
g(r)= & 2.114073 \times 10^{-3}+3.818921 \times 10^{-3} r-2.131285 \\
& \times 10^{-7} r^{2},
\end{aligned}
$$

where $r$ is the radius in $\mathrm{km}$. A similar fit to $\rho(r)$ to derive the pressure leads to a polynomial expression for pressure (in GPa) of

$$
\begin{aligned}
P(r)= & 3.853676 \times 10^{2}-3.083633 \times 10^{-2} r-1.184717 \\
& \times 10^{-5} r^{2}
\end{aligned}
$$

again with radius $r$ in $\mathrm{km}$.
Wavespeeds in the outer core are closely approximated by adiabatic self-compression (Dziewonski and Anderson 1981). While PREM does not specify a core temperature, with a CMB reference temperature $\left(T_{0}\right)$ it can be projected down the adiabat using PREM data. Using the relation $T(h)=T_{0} \exp \left(\gamma \int_{0}^{h} g(z) / \mathrm{V}_{\Phi}^{2}(z) d z\right)$ (Stacey and Davis 2008), the pressure, density, and temperature at any depth $h$ below the CMB may be calculated. Alfè et al. (2002) showed that the Grüneisen parameter $\gamma$ is virtually constant throughout the core and has the value 1.52 that I use to calculate normal core temperatures. However, various shock wave studies and empirical models suggest a range of $1.3 \leq \gamma \leq 1.8$ (Stacey and Davis 2008) so this is also investigated. I use a CMB temperature of 4,300 K throughout, except where the explicit thermal wavespeed variation is explored.

\section{Methods used}

There are various flavors of the hard sphere model proposed by different researchers. I focus on two properties of these models to apply them to the core. Each theoretical variant has an expression for the compressibility factor $Z=P /(\rho R T)$, essentially providing the equation of state for the liquid. $P$ (pressure), $T$ (absolute temperature), and $R$ (the universal gas constant) have their customary meanings, but the density $\rho$ is in moles per unit volume. With a CMB temperature, all of these can be calculated from PREM. $Z$ is related through the theory to the hard sphere diameter $\sigma$ and thence to the hard sphere packing fraction $\eta=\pi n \sigma^{3} / 6 . n$ is the number density, the number of particles per unit volume, related through the molecular weight of the liquid $M$ to the PREM density.

The second property is the liquid wavespeed. This is fixed by the hard sphere packing fraction $\eta$ and other free parameters of the particular theory variant. For each variant I search over the free parameters to determine the best-fitting set that reproduces PREM wavespeeds throughout the core. There are usually one or two free parameters to be searched over, so the best combination is determined computationally by a relatively undemanding grid search or line minimization. The following sections introduce the particular hard sphere theory variants.

\section{Carnahan-Starling hard sphere liquid}

The hard sphere model relates the compressibility factor $Z$ to the hard sphere packing fraction $\eta$ through a very simple formula. The best formulation of the hard sphere equation of state is Carnahan and Starling's (1969) one, wherein

$$
Z=\left(1+\eta+\eta^{2}-\eta^{3}\right) /(1-\eta)^{3} .
$$

Thus if $Z$ is known, $\eta$ may be solved for (provided $Z \geq 1$; clearly, $0 \leq \eta \leq 1)$ and $\sigma$ obtained. 
The hard sphere packing fraction also governs the wavespeeds in the hard sphere liquid. Rosenfeld (1999) derived the sound speed $c$ in the hard sphere liquid in terms of $Z$ (given by (3)), $\eta$ and the molecular weight $M$ :

$$
c^{2}=\frac{R T}{M}\left[Z+\eta \frac{d Z}{d \eta}+\frac{2}{3} Z^{2}\right] .
$$

The only free parameter in this theory is the mean atomic weight, $M$.

The expression appears to indicate that $c$ monotonically increases with $T$ (and decreases with $M$ ), but this is not true because $Z$ depends on $T$ as well. Plotting $c(T) / c\left(T_{m}\right)$ ( $T_{m}$ is the melting temperature, defined for a hard sphere liquid when $\eta=0.494$ ) in Fig. 1, one sees the surprising result that $c$ drops with decreasing $\eta$ (increasing $\mathrm{T}$ ) but then rises again as $\eta$ decreases ( $T$ continues to increase). Thus the dependence on $T$ is not straightforward and captures the behavior of both a liquid (wavespeed decreases with $T$ at high $\eta$ ) and a gas (wavespeed increases with $T$ at low $\eta$ ). For later use, it is helpful to define the quantity $p(\eta)=\left(1+\eta+\eta^{2}-\eta^{3}\right) /(1-\eta)^{3}$ and its derivative with respect to $\eta, p^{\prime}(\eta)=2\left(2+2 \eta-\eta^{2}\right) /(1-\eta)^{4}$.

\section{Hard sphere liquid mixtures}

The core is impure and can be viewed as a liquid mixture of different alloying elements whose sizes and masses differ from iron. Mansoori et al. (1971) extended the hard

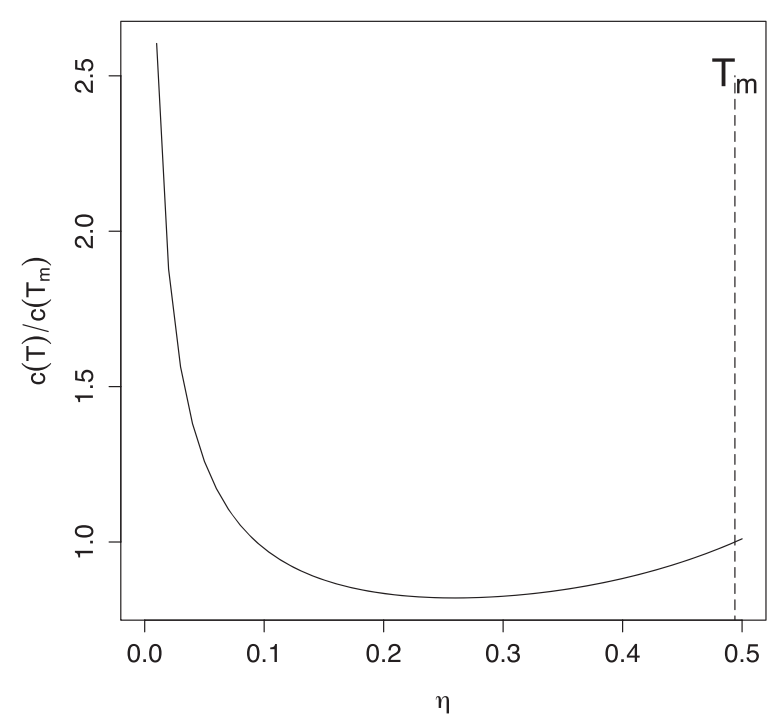

Fig. 1 Hard sphere liquid properties. Variation of wavespeed in hard sphere liquid $c$ with hard sphere packing fraction $\eta$. The speed is relative to the value at the melting temperature $\left(T_{m}\right)$, where there is a phase change in the liquid to solid form (at $\eta=0.494$ ). While Equation (4) suggests that the dependence should be monotonic on temperature (lower $\eta$ implies lower density thus higher $T$ ), the co-variation of $Z$ on $T$ leads to the more complex trend depicted. After Rosenfeld (1999) sphere liquid model to include mixtures by adding further terms to the expression for $Z$ :

$$
Z=\left[\left(1+\eta+\eta^{2}\right)-3 \eta\left(y_{1}+y_{2} \eta\right)-y_{3} \eta^{3}\right] /(1-\eta)^{3} .
$$

The $y_{1,2,3}$ terms depend on the mixture properties. If $x_{i}$ is the mole fraction of component $i$ in the $m$-component mixture with $\sum_{i=1}^{m} x_{i}=1$, then

$$
\begin{aligned}
\eta_{i} & =\frac{\pi}{6} n \sigma_{i}^{3} x_{i}, \quad \eta=\sum_{i=1}^{m} \eta_{i} \\
y_{1} & =\sum_{j>i=1}^{m} \Delta_{i j}\left(\sigma_{i}+\sigma_{j}\right)\left(\sigma_{i} \sigma_{j}\right)^{-1 / 2} \\
y_{2} & =\sum_{j>i=1}^{m} \Delta_{i j} \sum_{k=1}^{m}\left[\frac{\eta_{k}}{\eta}\right] \frac{\left(\sigma_{i} \sigma_{j}\right)^{1 / 2}}{\sigma_{k}}, \\
y_{3} & =\left[\sum_{i=1}^{m}\left[\frac{\eta_{i}}{\eta}\right]^{2 / 3} x_{i}^{1 / 3}\right]^{3}, \\
\Delta_{i j} & =\left[\left(\eta_{i} \eta_{j}\right)^{1 / 2} / \eta\right]\left[\left(\sigma_{i}-\sigma_{j}\right)^{2} /\left(\sigma_{i} \sigma_{j}\right)\right]\left(x_{i} x_{j}\right)^{1 / 2}
\end{aligned}
$$

For a single component liquid, $\Delta_{i i}=0$ implying that $y_{1}=y_{2}=0$, and $y_{3}=1$, reducing Equation (5) to (3). In a two-component liquid, the theory's free parameters are therefore the size ratio (relative to iron) and mass of the particle. Assuming a weight percentage of a light element in the core, these factors determine the mole fractions $x_{i}$ and the mean atomic weight $M$ in the liquid.

\section{Transition metal hard sphere liquids}

Yokoyama (2001a) showed that the hard sphere model could be successfully applied to calculate the sound velocities in liquid metals at 1 atm close to their melting temperatures. This was achieved by modifying Rosenfeld's (1999) expression for the wavespeed dependence on packing fraction to account explicitly for the dependence of hard sphere diameter on temperature. In addition, Yokoyama (2001a) recognized that the electronic structure of the transition metals added a suite of longer-ranged but weak repulsive forces to the inter-particle interactions in the liquid. These two factors lead to modifications to the expression for wavespeeds. Including the hard sphere size dependence on $T$ changes Equation (4) to

$$
\begin{gathered}
c^{2}=\frac{R T}{M}\left[p(\eta)+\eta p^{\prime}(\eta)+\frac{2}{3}\left[3 \eta p^{\prime}(\eta)(\partial \log \sigma / \partial \log T)_{V}\right.\right. \\
\left.+p(\eta)]^{2}\right] .
\end{gathered}
$$


If ( $(\partial \log \sigma / \partial \log T)_{V}=0$, Equation (4) is recovered. The explicit temperature dependence of $\sigma$ given by Yokoyama (2001a) is $(\partial \log \sigma / \partial \log T)_{V}=-k_{1} \sqrt{T / T_{m}} /\left(1-k_{2}\right.$ $\left.\sqrt{T / T_{m}}\right)$ with constants $k_{1}=0.0444$ and $k_{2}=$ 0.112 . However, for the hard sphere liquid, all properties should be relatable to $\eta$, and the explicit $T$ dependence is only valid at a particular pressure, in this case $1 \mathrm{~atm}$. Noting that $(\partial \log \sigma / \partial \log T)_{V}=(T / \sigma)$ $(d \sigma / d \eta)_{V}(d \eta / d Z)_{V}(d Z / d T)_{V}$, an expression only involving $\eta$ may be obtained: $(\partial \log \sigma / \partial \log T)_{V}=-p(\eta) /$ $\left(3 \eta p^{\prime}(\eta)\right)$. Substituting this into (7) eliminates the squared term and lowers wavespeeds. Rosenfeld (1999) shows this term is $(\partial P / \partial T)_{V}$. Using the Maxwell relation for $V(P, T),(\partial P / \partial T)_{V}=\alpha K_{T}$ which, if zero, implies that the thermal expansivity $\alpha$ is zero - unlikely for the core or indeed any substance in a planetary interior (Helffrich and Connolly 2009). Thus the $\eta$-only dependence through $Z$ is unphysical and implies that a viable wavespeed model must contain factors leading to a nonzero thermal expansivity.

The electronic contributions to the wavespeed are an electron-gas energy (arising from the chargecompensating valence electrons in the liquid), the electrostatic repulsive energy of the charged ionic cores, and a $s-d$ orbital hybridization term $\left(B_{H}\right)$ due to the irregular filling of the $d$ orbitals among the transition metals. See Shimoji (1977) and Yokoyama (2001b) for a discussion of these terms. $B_{H}$ is essentially a static correction to $Z$ for a liquid for a particular metallic species (for the core, iron). Thus

$$
\begin{gathered}
B_{H}=\frac{a_{m}^{6}}{6} \epsilon_{\text {Ryd }}\left[0.031 z+\left(0.916 z^{4 / 3}+1.8 z^{2}\right) / a_{m}-4.42 z^{5 / 3} / a_{m}^{2}\right] \\
-3 k_{B} T_{m}\left(1+\eta_{m}+\eta_{m}^{2}-\eta_{m}^{3}\right) /\left(1-\eta_{m}\right)^{3} .
\end{gathered}
$$

$k_{B}$ is Boltzmann's constant and $\epsilon_{\mathrm{Ryd}}$ is the Rydberg energy, $2.1798741 \times 10^{-18} \mathrm{~J} . a_{m}$ is the hard sphere radius at melting conditions nondimensionalized by dividing it by the Bohr length $\left(5.2917725 \times 10^{-11} \mathrm{~m}\right)$, and $\eta_{m}$ is the hard sphere packing fraction at melting (here taken to be 0.463 in contrast to Rosenfeld's value). The particular metal is characterized by a valence electron count $z$, melting temperature $T_{m}$ and melting density $\rho_{m}$ (and number density $n_{m}$ ) defining the hard sphere radius through $\eta_{m}=$ $4 / 3 \pi a_{m}^{3} n_{m}$. For iron, the valence in the liquid metal state is $z=1.33$ (Yokoyama 2001a).

Incorporating the electron gas $\left(u_{\mathrm{eg}}\right)$ and the ion repulsion $\left(u_{\text {ion }}\right)$ contributions to the compressibility factor allows the hard sphere packing fraction $\eta$ and WignerSeitz radius $a$ to be determined. $u_{\mathrm{eg}}, u_{\mathrm{ion}}$, and $B_{H}$ all serve to increase the effective $Z$, given by

$$
\begin{aligned}
Z= & -\left[\epsilon_{\mathrm{Ryd}}\left[0.031 z+\left(0.916 z^{4 / 3}+1.8 z^{2}\right) / a-4.42 z^{5 / 3} / a^{2}\right]-6 B_{H} / a^{6}\right] \\
& /\left(3 k_{B} T\right)+\left(1+\eta+\eta^{2}-\eta^{3}\right) /(1-\eta)^{3} .
\end{aligned}
$$

With $\eta$ (and $a$, again in multiples of the Bohr radius) obtained, the liquid metal wavespeed, incorporating (7), is

$$
\begin{aligned}
c^{2}= & \frac{R T}{M}\left[\left(\epsilon_{\mathrm{Ryd}}\left[u_{\mathrm{eg}}+u_{\mathrm{ion}}\right]+B_{H} / a^{6}\right) /\left(k_{B} T\right)+p(\eta)+\eta p^{\prime}(\eta)\right. \\
& \left.+\frac{2}{3}\left[3 \eta p^{\prime}(\eta)(\partial \log \sigma / \partial \log T)_{V}+p(\eta)\right]^{2}\right],
\end{aligned}
$$

with $u_{\mathrm{eg}}=-0.031 z / 3-(4 / 9)\left(0.916 z^{4 / 3}+1.8 z^{2}\right) / a$ and $u_{\text {ion }}=(22.1 / 9) z^{5 / 3} / a^{2}$. The electronic terms serve to raise wavespeeds relative to liquid without charge interactions both by increasing $\eta$ for a given $Z$ and by adding charge-related terms $\left(u_{\mathrm{eg}}, u_{\mathrm{ion}}\right.$, and $\left.B_{H}\right)$ to (7). Within this theoretical framework for a particular metal, the free parameters governing liquid speed are valence electron count $z$ and mean atomic weight $M$.

\section{The Lennard-Jones hard sphere liquid with attractive forces}

A characteristic property of any liquid is its tendency to cohere and to maintain a fixed volume in the absence of confinement. A simple way to parameterize this is by defining an intermolecular potential based on separation $r, u(r)$, whose gradient entails both repulsive and attractive forces. The Lennard-Jones potential is a common one used for this parameterization, given by

$$
u(r)=4 \epsilon\left[(\sigma / r)^{12}-(\sigma / r)^{6}\right]
$$

(Fig. 2). $\epsilon$ is an interaction energy and $\sigma$ is a length scale called the collision diameter. There are various ways to incorporate an interaction potential into a liquid theory, but for simplicity I choose Kolafa and Nezbeda's (1994) parameterization based on the Barker and Henderson (1976) recipe for determining hard sphere size in the liquid. Again, the hard sphere diameter is chosen by matching $Z$, given in this theory by

$$
Z=p(\eta)+\rho^{*}\left(1-2 \gamma \rho^{*}\right) \exp \left(-\gamma \rho^{* 2}\right) \Delta B_{2}(T)+f\left(T, \rho^{*}\right) .
$$

$\rho^{*}=n \sigma^{3}$ is the reduced number density, and $\eta=$ $\pi \rho^{*} / 6$. Kolafa and Nezbeda (1994) give polynomial expansions in powers of $T^{1 / 2}$ and $\log T$ for $\Delta B_{2}(T)$ and additionally powers of $\rho^{*}$ for $f\left(T, \rho^{*}\right) . \gamma=1.9290728$ is an empirical fitting constant. The liquid wavespeed is given by Equation (7), with $d Z / d \eta\left(=p^{\prime}(\eta)\right)$ and $(\partial \log \sigma / \partial \log T)_{V}$ evaluated numerically. Thus the free parameters in the method are the Lennard-Jones energy scale $\epsilon$ and $M$, the mean atomic weight of the liquid. 


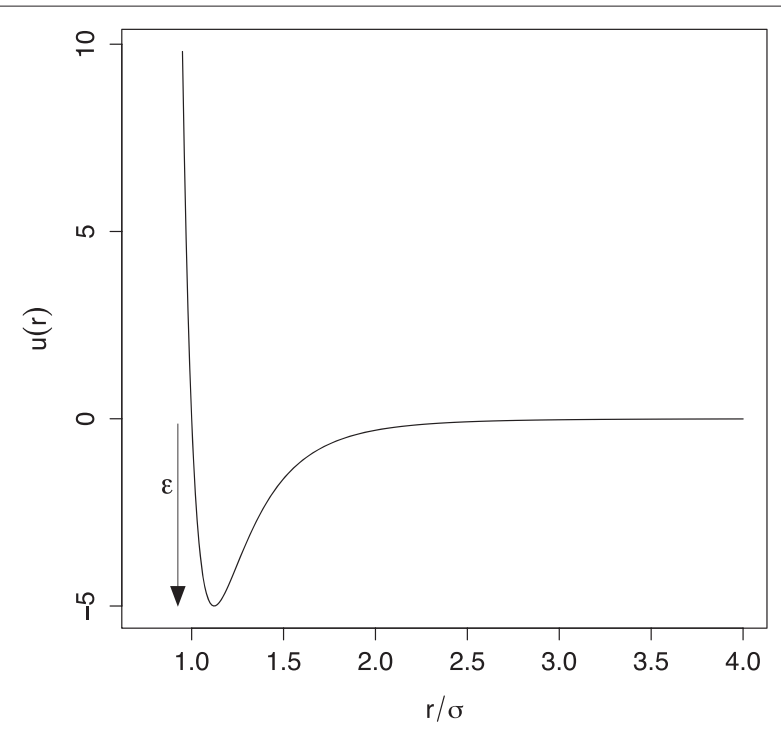

Fig. 2 Lennard-Jones potential. Lennard-Jones potential with $\epsilon=5$. The potential scale governs the depth of the well at the minimum $r / \sigma=2^{1 / 6}$, beyond which the force between liquid constitutents is attractive. The function tends to zero at infinite separation and to infinitely strong repulsion at zero separation

\section{Results}

\section{Carnahan-Starling hard sphere liquid}

The only free parameter in this theory is the molecular weight of the liquid. The seismic wavespeed is calculated with Equation (4). Minimizing the misfit to PREM wavespeeds by varying $M$ leads to a best-fit $M=$ $6.1 \mathrm{~g} \mathrm{~mol}^{-1}$. The comparison with PREM (Fig. 3) shows the fit not only to be poor but leads to an unreasonably low molecular weight for the core. The model is evidently too simple to be a realistic description for the Earth. Equation (7) is an alternative expression for the hard sphere liquid wavespeed when there is an explicit temperature dependence to $\sigma$. If in the spirit of the hard sphere model this dependence is parameterized in terms of $\eta$, then a satisfactory fit may be obtained. However, parameterizations in $\eta$ lead to $d c / d T>0$ which is unphysical for the larger $\eta$ values found in the core (Fig. 1). To avoid this I parameterize the correction in powers of $\sqrt{T}$ as did Protopapas et al. (1973). The explicit form for the correction is in rational polynomial form, parameterized with numerator and denominator coefficients $c_{i}$ and $d_{i}$ :

$$
(\partial \log \sigma / \partial \log T)_{V}=\left(c_{0}+c_{1} \sqrt{T}+c_{2} T\right) /\left(1+d_{1} \sqrt{T}+d_{2} T\right)
$$

(Figure 3). This correction is entirely an expedient tactic whose coefficient estimates come from fitting velocity

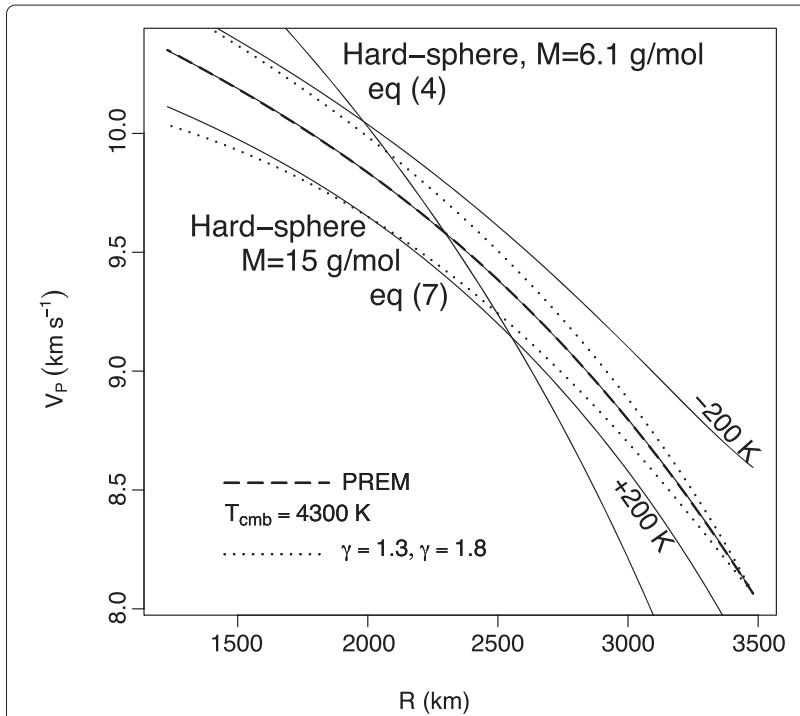

Fig. 3 Hard sphere liquid performance. Optimized hard sphere liquid wavespeeds for the Carnahan-Starling model compared with PREM The best-fit to PREM, using Equation (4) for the wavespeed, is for a liquid atomic weight of $M=6.1 \mathrm{~g} \mathrm{~mol}^{-1}$. If $(\partial \log \sigma / \partial \log T)_{V}$ is used with Equation (7) for the wavespeed, a much better fit results virtually overlying PREM. Equation (13) parameterizes $(\partial \log \sigma / \partial \log T)_{V}$ with $\left(c_{0}, c_{1}, c_{2}\right)=\left(-1.170076 \times 10^{-1}, 3.297547 \times 10^{-3}, 2.274412 \times 10^{-5}\right)$ and $\left(d_{1}, d_{2}\right)=(-0.0294468508,0.0002102121)$. The lines paralleling PREM show the wavespeeds for $\pm 200 \mathrm{~K}$ change in $T_{\mathrm{cmb}}$. Dotted lines show result of varying $\gamma$ through the range shown. The result is similar to varying $T_{\mathrm{cmb}}$ and a corresponding change to $(\partial \log \sigma / \partial \log T)_{V}$

residuals when $M=15 \mathrm{~g} \mathrm{~mol}^{-1}$ to (13). It shows how good a hard sphere model can be if it can properly predict this dependence, however.

The applicability of the Carnahan-Starling (CS) liquid model to the core is severely limited. Fig. 4 depicts those limits, which derive from the liquid's molecular weight. Only low molecular weight liquids $\left(M<20 \mathrm{~g} \mathrm{~mol}^{-1}\right)$ are admissible throughout the core.

\section{Hard sphere liquid mixtures}

The free parameters in this model are the mass ratio and the radius ratio with respect to iron (at a fixed $10 \mathrm{wt} \%$ light element). A light element mass $m$ at a fixed weight percent in the core fixes the mole fraction and thus $M$ for the bulk liquid. A grid search over $1 \leq m \leq 56$ and ratio $0.2 \leq r \leq 1$ (corresponding to a range of element radii from He through $\mathrm{H}$ to Fe (Cordero et al. 2008)) yields a bestfit to PREM with $r=0.25$ and $m=1.45 \mathrm{~g} \mathrm{~mol}^{-1}$. Fig. 5 shows the resulting fit, which is poor at the base of the core when $\eta$ approaches freezing conditions for the hard sphere liquid. This model suggests that the core's light element is dominantly hydrogen, surprisingly affirmed by the radius ratio for $\mathrm{H} / \mathrm{Fe}$ : $0.31 / 1.32=0.235$ (Cordero et al. 2008). 


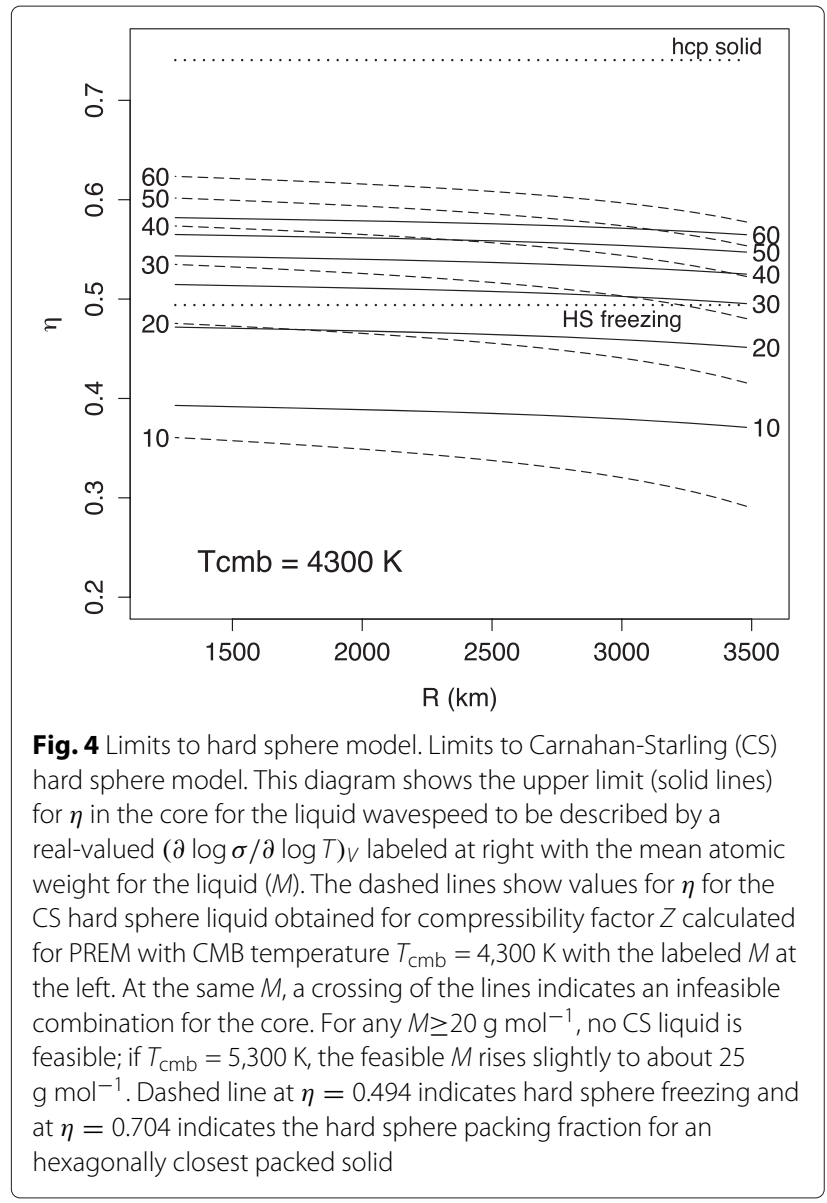

\section{Transition metal hard sphere liquids}

The free parameters in this model are the number of valence electrons $z$ and the liquid molar mass $M$. The grid search is over $1 \leq z \leq 2.5$ and $1 \leq M \leq 56$. The best-fit combination is $z=1.649$ and $M=48.8 \mathrm{~g} \mathrm{~mol}^{-1} . z$ is slightly higher than the $1 \mathrm{~atm}$ value at melting $(z=1.33)$. Fig. 6 shows that the model liquid properties closely follow PREM through straight application of the theory. The average deviation from PREM throughout the core is $0.27 \%$; this is only slightly larger than the approximately $0.25 \%$ wavespeed deviations found at the top of the outer core (Helffrich and Kaneshima 2010; Kaneshima and Helffrich 2013). It is possible to improve the fit by allowing a radial dependence for $z$ and $M$, but I defer this to the discussion of this particular model's adjustment to decrease speeds at the top of the core. This theory prescribes only a weak temperature dependence for the wavespeeds.

The Lennard-Jones hard sphere liquid with attractive forces The free parameters in this model are the energy scale $\epsilon$ and the liquid molar mass $M$. A grid search over the typical $1 \leq M \leq 56$ and $1 \mathrm{~K} \leq \epsilon / k_{B} \leq 5,000 \mathrm{~K}$ allows for two
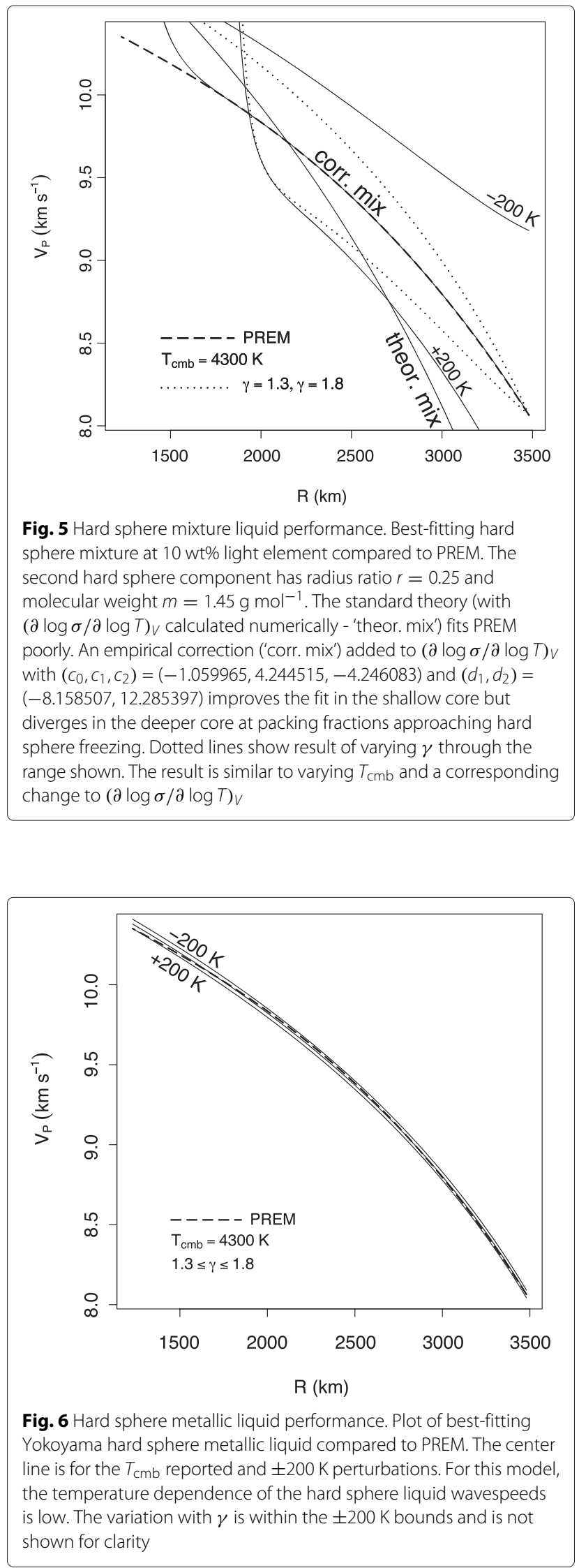
solutions. The first is a low- $\epsilon$ solution with $\epsilon / k_{B}=20$ $\mathrm{K}$ and $M=6.1 \mathrm{~g} \mathrm{~mol}^{-1} . \epsilon$ is so small that this is effectively no attraction at all at core temperatures, and is similar to the unmodified CS hard sphere model. The other solution has strong attractive forces with $\epsilon / k_{B}=$ $2,460 \mathrm{~K}$ and $M=6.5 \mathrm{~g} \mathrm{~mol}^{-1}$. The comparison to PREM is shown in Fig. 7. The mean deviation from PREM is $0.84 \%$, but the radial trend is much flatter than PREM. This is a poor fit with the unmodified theory, but an empirical temperature-dependent correction can improve it in the shallower parts of the outer core.

\section{Discussion}

With approximate fits of each model to the core's structure, we can ask what perturbations to it will approximate the observed wavespeed reductions from PREM. Fig. 8 shows the perturbations to individual models. The models, for comparison, are KHOCQ (Helffrich and Kaneshima 2010) and KHOMC (Kaneshima and Helffrich 2013). They deviate from PREM in the topmost approximately $350 \mathrm{~km}$ of the outer core by approximately $0.25 \%$.

\section{The hard sphere model}

The simplest model to evaluate is the hard sphere model (Fig. 1a). The only adjustable parameter in the theory is the mass $M$ of the particle. Additionally, the temperature may be varied to allow conditions to deviate from adiabatic. The variation of each of these is shown in Fig. 8 by two virtually coincident lines deviating from the fitted model (see Fig. 3) in the topmost $375 \mathrm{~km}$ of the core. One case shows an increasingly subadiabatic (hotter) temperatures reaching about $17 \mathrm{~K}$ higher than the adiabat. This is a surprisingly small value but suggests that the heat flux would not be significantly reduced down the adiabat: by only approximately $4 \%$. Alternatively, only a $2 \%$ decrease in density of the core material is needed to reduce the wavespeeds there. The mass and temperature perturbations are both approximately linear, so any linear combination of mass and temperature within these limits could also serve.

However, the reduction is from $M=15 \mathrm{~g} \mathrm{~mol}^{-1}$, an already extremely light core. For this $M$ to represent a 10 wt\% light element mix, the light element mass would be a combination of $\mathrm{H}+\mathrm{He}$ and the concentration would be around $79 \mathrm{~mol} \%$. This is quite a large amount of hydrogen

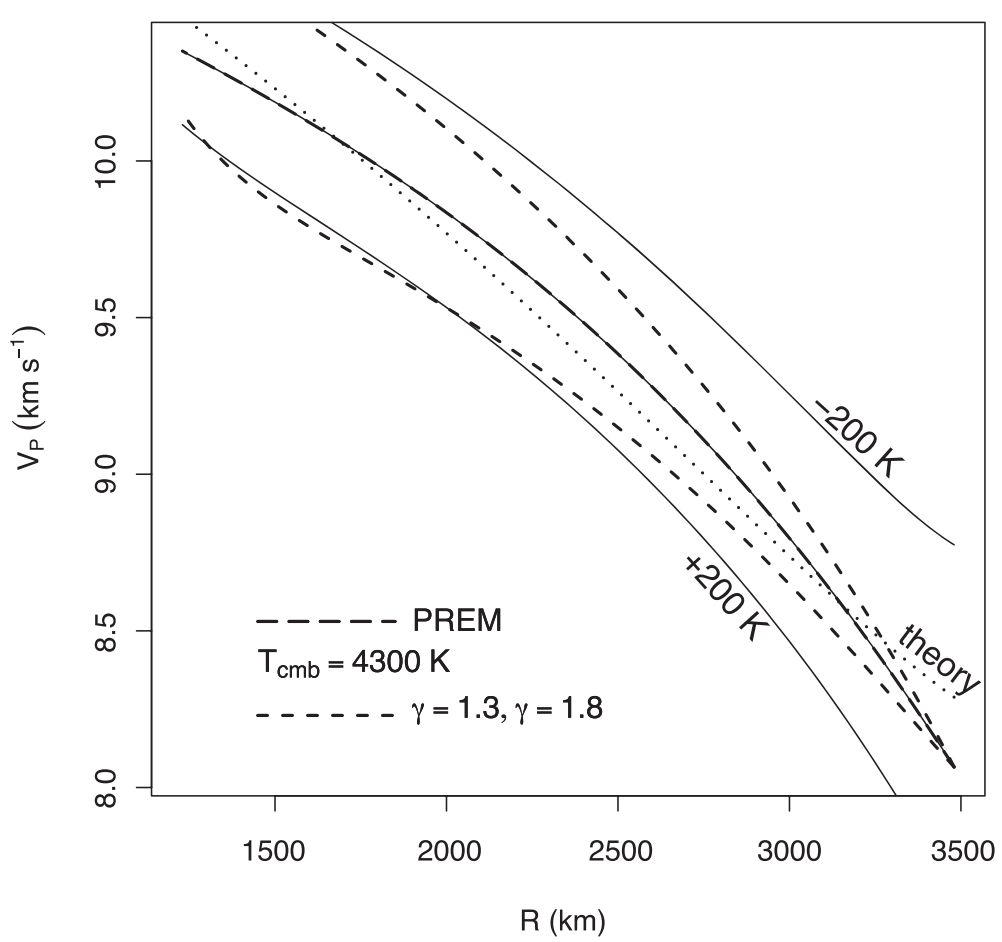

Fig. 7 Lennard-Jones model performance. Plot of best-fitting Lennard-Jones hard sphere liquid compared to PREM. The dotted line ('theory') is the fit to the unmodified theory with $\epsilon / K_{B}=2640 \mathrm{~K}$ and $M=6.5 \mathrm{~g} \mathrm{~mol}^{-1}$. The solid lines show an augmented model with a temperature dependent correction to $(\partial \log \sigma / \partial \log T) V$ with $\left(c_{0}, c_{1}, c_{2}\right)=\left(-7.928909 \times 10^{-2}, 1.887171 \times 10^{-3},-1.108079 \times 10^{-5}\right)$ and $\left(d_{1}, d_{2}\right)=\left(-3.06458231 \times 10^{-2}\right.$, $2.259932 \times 10^{-4}$ ) in addition to the numerical differentiation of $\sigma$. The center line is for the reported $T_{c m b}$ and $\pm 200 \mathrm{~K}$ perturbations. PREM is effectively overlaid by the center solid line. Dotted lines show result of varying $\gamma$ through the range shown. The result is similar to varying $T_{c m b}$ and a corresponding change to $(\partial \log \sigma / \partial \log T)_{V}$ 


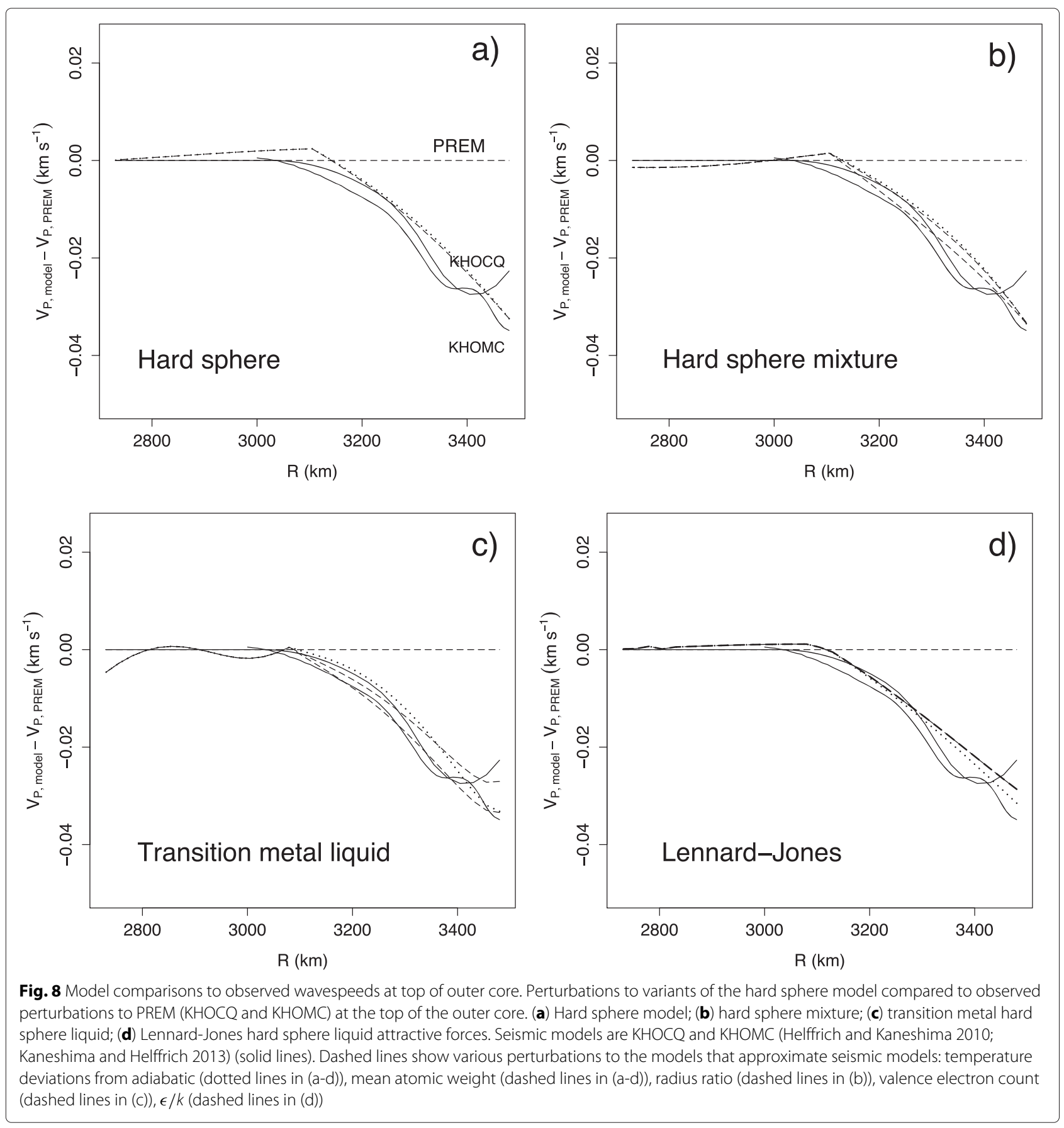

and helium given the expected core composition based on chondritic element abundances (Allègre et al. 1995), leaving no possibility of others whose presence is more likely on cosmochemical grounds.

\section{The hard sphere mixture}

The adjustable parameters in this model are radius and mass ratio relative to iron, and temperature. A hard sphere mixture is subject to an even smaller amount of subadiabatic temperature increase than a singlecomponent hard sphere fluid, only about $10 \mathrm{~K}$. The wavespeed decrease could also arise by a $3 \%$ increase in radius ratio relative to the reference ratio, 0.25 , or an increase to 0.2575 . Based on covalent radii for light elements, this represents a species change to $\mathrm{Na}$ or $\mathrm{Mg}$ among period 3 elements or $\mathrm{Cr}$ and $\mathrm{Mn}$ in period 4 elements: all others are significantly larger or smaller (Cordero et al. 2008). The mass must decrease, however, 
by about $1 \%$, restricting the elements to $\mathrm{Cr}$ or $\mathrm{Mn}$. The variations are again nearly linear, so linear combinations of any three of the factors could work to reduce wavespeeds in the core. However, the low $m$ also suggests that the core is dominantly an $\mathrm{H}+\mathrm{He}$ mix, unlikely for cosmochemical reasons as before.

\section{The transition metal hard sphere liquid}

This is the most intriguing of the models because it is a very good description of outer core wavespeeds all by itself (Fig. 6). The adjustable parameters are the $M$ and $z$, along with $T$. The best-fitting values unfortunately yield the largest differences to PREM at both the top and base of the core, where the structural variations are most interesting. The basic model (Equation (10)) is therefore perturbed to minimize the difference to PREM by adding a radius dependence to the valence electron number $\delta z$ to fit PREM, given by

$$
\delta z(h)=\left\{\begin{array}{cl}
\frac{c_{0}+c_{1} h+c_{2} h^{2}+c_{3} h^{3}}{1+d_{1} h+d_{2} h^{2}} & , h>400 \\
\frac{a_{0}+a_{1} h}{1+b_{1} h} & , h \leq 400
\end{array}\right.
$$

with $\left(c_{0}, c_{1}, c_{2}, c_{3}\right)=\left(-9.402105 \times 10^{-2}, 7.577652 \times\right.$ $\left.10^{-4},-1.62307 \times 10^{-6}, 1.039725 \times 10^{-9}\right),\left(d_{1}, d_{2}\right)=$ $\left(-3.455461 \times 10^{-3}, 5.173742 \times 10^{-6}\right)$ and $\left(a_{0}, a_{1}\right)=$ $(-0.0958702639,0.0005057239)$ and $b_{1}=0.004435071(h$ is in $\mathrm{km}$ in these expressions). This is admittedly overfitting PREM, but it leads to a simple, linear perturbation to the layer density in the outermost core shown in Fig. 9. The $M$ decrease along with an adiabat is about $1.74 \%$. At the same time, the valence electronic charge $z$ decreases by $8 \%$ from 1.686 to 1.553 . An unchanged $z$ and $M$ requires much higher temperatures to slow the layer due to the weak theoretical temperature dependence (Fig. 9): the topmost core is virtually $400 \mathrm{~K}$ hotter than the projection of a 4,300 $\mathrm{K}$ adiabat from $400 \mathrm{~km}$ deep in the core. This means that there is only a $5 \mathrm{~K}$ temperature drop throughout the topmost $400 \mathrm{~km}$ of the core. Hence there would be a low core heat flux, only $1.2 \%$ of the adiabatic one. This could contribute to solving the paradox of the young inner core age compared to the oldest recorded magnetic field (Gomi et al. 2013; Labrosse et al. 2001).

The model, moreover, provides a sensible atomic mass for the core liquid, $48.8 \mathrm{~g} \mathrm{~mol}^{-1}$. If this represents a 10

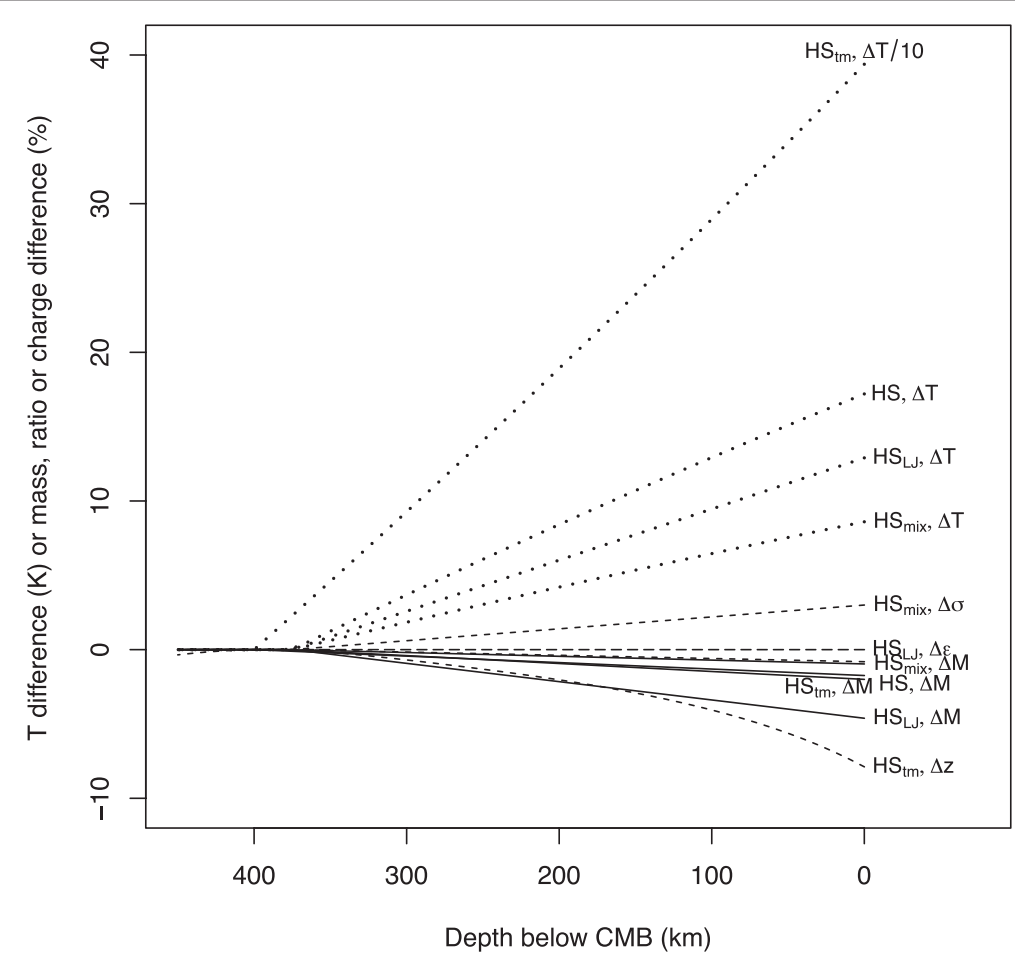

Fig. 9 Summary of perturbations to conditions at top of outer core. Perturbations to conditions at the top of the core to approximately fit the observed velocity perturbations to PREM for various hard sphere model variants: straight hard sphere $(H S)$, hard sphere mixture $\left(H S_{\text {mix }}\right)$, a hard sphere liquid with electronic structure $\left(\mathrm{HS}_{\mathrm{tm}}\right)$, and a hard sphere liquid with attractive forces $\left(\mathrm{HS}_{\mathrm{L}}\right)$. Changes to temperature $(\Delta T)$, hard sphere diameter $(\Delta \sigma)$, density $(\Delta M)$, valence electron charge $(\Delta z)$, or interaction potential $(\Delta \epsilon)$ are shown. $\Delta T$ is the temperature difference relative to the adiabatic temperature at the depth below the CMB. The other perturbations to $\sigma, M, z$, or $\epsilon$ are to the reference value yielding PREM (Figs. 3, 5, 6 and 7). Most hard sphere variants require only percent-level changes to intrinsic parameters or few-K changes to temperature to reduce wavespeeds at the top of the core, except for the transition metal hard sphere liquid 
wt\% light element mix with iron, it maps into about a 20 mol\% mixture of an element whose atomic mass is about $25 \mathrm{~g} \mathrm{~mol}^{-1}$. This puts it squarely in the range between $\mathrm{C}$, $\mathrm{N}, \mathrm{O}$ (on the light side - 12 to $16 \mathrm{~g} \mathrm{~mol}^{-1}$ ) and $\mathrm{Si}$ and $\mathrm{S}$ (on the heavy side - 28 to $32 \mathrm{~g} \mathrm{~mol}^{-1}$ ). Thus it is cosmochemically plausible for a quasi-chondritic Earth model. Allègre et al. (1995) suggest that there are 7.35 wt\% Si, 2.3 $\mathrm{wt} \% \mathrm{~S}$, and $4.1 \mathrm{wt} \% \mathrm{O}$ in the core, which, normalized to an average atomic weight, is $23 \mathrm{~g} \mathrm{~mol}^{-1}$, close to the value prescribed by this hard sphere model variant.

\section{The Lennard-Jones hard sphere liquid}

The adjustable parameters in this model are the energy scale $\epsilon$ and the atomic mass $M$, along with temperature. There could be a mass decrease of about $3 \%$ relative to the deeper core near the top. Alternatively, there could be a slight decrease, by $1 \%$, to the energy scale. This would mean that the alloying element slightly reduces the intermolecular attraction between species in the liquid, but it is difficult to attribute it to any particular element's intrinsic chemical properties such as its position in the periodic table. Temperature, on the other hand, is only $10 \mathrm{~K}$ higher than the adiabatic gradient, leading to slightly subadiabatic conditions and a $2.5 \%$ lower heat flux than what is conducted down the adiabat. On the other hand, the mean atomic mass is $6.5 \mathrm{~g} \mathrm{~mol}^{-1}$, implies that the core's light element is pure hydrogen, with a mole fraction of $83 \%$. This is again in conflict with cosmochemical expectations for the core's composition because it excludes any other element from being in the core (Allègre et al. 1995).

\section{Summary and analysis}

Table 1 summarizes the performance of the models analyzed here. Though all hard sphere model variants may be made to fit the wavespeeds in the outer core, only one fits and provides a plausible view of the core from which sensible deviations may be explored: the transition metal hard sphere liquid.

The main reason why most variants fail is because hard sphere models use as a reference state the ideal gas and add further Virial-like terms in powers of density that capture more complex interactions between liquid particles. Dense liquids like the core are quite far from the ideal gas reference and therefore require many terms before their behavior can be completely expressed. The CarnahanStarling model actually captures Virial coeffients up to order 9 (Hansen and McDonald 2013), but this is clearly insufficient (Fig. 3). It serves as the basic reference for all of the hard sphere variants, so all are limited by the need for further higher order terms.

The transition metal liquid model recognizes additional repulsive forces that change the reference model to something other than an ideal gas. The particles have longerranged repulsive interactions that are essentially electrostatic. These act to increase wavespeeds relative to other models at a given $Z$ (Equations (7), (9), and (10)). Hence this model leads to higher liquid mean atomic weights to counterbalance the additional force contributions and to match the wavespeeds in the core.

\section{Conclusions}

The hard sphere model for liquids may successfully be applied to the core. The standard versions - a hard sphere liquid with only repulsive forces, a liquid mixture of hard spheres, and a hard sphere liquid with attractive forces - require the addition of an explicit temperature dependence to the hard sphere diameter, but they can fit the uppermost outer core well. Among these, the mixedsphere liquid performs worst near the base of the core where packing fractions are highest. All, however, suffer from the defect that the mean atomic weight of the core liquid is too low for any cosmochemically plausible composition because they require so much hydrogen and helium match core wavespeeds, leaving little room in the core for the heavier light elements that have sensible cosmochemical constraints (Allègre et al. 1995).

The best hard sphere model for the core is a transition metal liquid whose valence electrons form an electron sea bathing and charge-compensating the spherical ionic cores. This model yields a surprisingly good

Table 1 Summary of hard sphere model performance reproducing PREM

\begin{tabular}{|c|c|c|c|}
\hline $\begin{array}{l}\text { Model variant } \\
\text { (symbol) }\end{array}$ & Comments & Adjustments & Plausibility \\
\hline Carnahan-Starling (HS) & $d V / d r$ discrepant & $\begin{array}{l}\text { Additional explicit thermal expansivity contri- } \\
\text { butions }\end{array}$ & $\begin{array}{l}\text { No. } M \text { too small; requires large } \\
\text { amounts of } \mathrm{H} \text { or } \mathrm{He} \text { in the core. }\end{array}$ \\
\hline $\begin{array}{l}\text { Hard sphere mixture } \\
\left(\mathrm{HS}_{\text {mix }}\right)\end{array}$ & $d V / d r$ discrepant & $\begin{array}{l}\text { Additional explicit thermal expansivity contri- } \\
\text { butions, but diverge at high } \eta \text { conditions at } \\
\text { depth }\end{array}$ & No. M too small. \\
\hline $\begin{array}{l}\text { Transition metal liquid } \\
\left(\mathrm{HS}_{\mathrm{tm}}\right)\end{array}$ & $\begin{array}{l}\text { Average deviation } 0.27 \% \text { from } \\
\text { PREM throughout core }\end{array}$ & None & $\begin{array}{l}\text { Yes. } M \text { slightly lower than Fe } \\
\text { atomic weight. }\end{array}$ \\
\hline $\begin{array}{l}\text { Lennard- Jones hard } \\
\text { sphere }\left(H S_{\sqcup J}\right)\end{array}$ & $\begin{array}{l}\text { Average deviation } 0.84 \% \text { from } \\
\text { PREM throughout core; } d V / d r \text { not } \\
\text { PREM-like }\end{array}$ & $\begin{array}{l}\text { Additional explicit thermal expansivity contri- } \\
\text { butions }\end{array}$ & No. M too small. \\
\hline
\end{tabular}


approximation to PREM wavespeeds throughout the core with a fixed number of valence electrons and atomic mass $\left(z=1.649\right.$ and $\left.M=48.8 \mathrm{~g} \mathrm{~mol}^{-1}\right)$. To mix $10 \mathrm{wt} \%$ of a light element with iron to yield this atomic mass, that element's mass must be about $25 \mathrm{~g} \mathrm{~mol}^{-1}$. This compares favorably to one estimate of the core's average light element mass, $23 \mathrm{~g} \mathrm{~mol}^{-1}$ (Allègre et al. 1995). Thus one model satisfies PREM wavespeed constraints and cosmochemical constraints on the core.

Using the hard sphere electronic liquid model to examine the wavespeed deviations from PREM at the top of the outer core, it appears that a approximately $1.7 \%$ decrease in $M$ at the top of the core (along with an $8 \%$ decrease in valence electrons $z$ ) can explain the wavespeed reduction there. With no change in $M$ or $z$, a linear temperature increase over the adiabat of approximately $400 \mathrm{~K}$ can also explain the wavespeed reduction. Therefore, a thermally and chemically stratified layer at the top of the core is feasible within the framework of the hard sphere model within these mass anomaly and temperature limits. Its success further suggests that the model may, in future, play an integrated role in thermal histories of the core and mantle (Hirose et al. 2013; Labrosse et al. 2001).

\section{Competing interests}

The author declares that he has no competing interests.

\section{Acknowledgements}

I thank the reviewers for their insightful comments that helped to improve the manuscript. I also thank John Hernlund for discussions about the consequences of layering in the core on its thermal evolution.

Received: 1 December 2014 Accepted: 20 April 2015

Published online: 21 May 2015

\section{References}

Alfè D, Price GD, Gillan MJ (2002) Iron under Earth's core conditions: liquid-state thermodynamics and high-pressure melting curve from ab initio calculations. Phys Rev B 65:165118

Allègre C, Poirier J-P, Humler E, Hofmann AW (1995) The chemical composition of the Earth. Earth Planet Sci Lett 134:515-526

Barker JA, Henderson D (1976) What is "liquid"? Understanding the states of matter. Rev Mod Phys 48:587-671

Birch, F (1952) Elasticity and constitution of the Earth's interior. J Geophys Res 57:227-286

Birch F (1964) Density and composition of the mantle and core. J Geophys Res 69:4377-4388

Carnahan NF, Starling KE (1969) Equation of state for nonattracting rigid spheres. J Chem Phys 51:635-636

Cordero B, Gómez V, Platero-Prats AE, Revés M, Echeverría J, Cremades E, Barragán F, Alvarez S (2008) Covalent radii revisited. Dalton Trans. 2008:2832-2838

Dehant V, Banerdt B, Lognonne P, Grott M, Asmar S, Biele J, Breuer D, Forget F, Jaumann R, Johnson C, Knapmeyer M, Langlais B, Feuvre ML, Mimoun D, Mocquet A, Read P, Rivoldini A, Romberg O, Schubert G, Smrekar S, Spohn T, Tortora P, Ulamec S, Vennerstrom S (2012) Future Mars geophysical observatories for understanding its internal structure, rotation, and evolution. Planet Space Sci 68:123-145

Dziewonski A, Anderson DL (1981) Preliminary reference Earth model. Phys Earth Planet Inter 25:297-356

Gomi H, Ohta K, Hirose K, Labrosse S, Caracas R, Verstraete MJ, Hernlund JW (2013) The high conductivity of iron and thermal evolution of the Earth's core. Phys Earth Planet Inter 224:88-103
Hansen J-P, McDonald IR (2013) Theory of simple liquids. Academic Press, Amsterdam

Helffrich G (2014) Outer core compositional layering and constraints on core liquid transport properties. Earth Planet Sci Lett 391:256-262

Helffrich G, Connolly JAD (2009) Physical contradictions and remedies using simple polythermal equations of state. Am Min 94:1616-1619

Helffrich G, Kaneshima S (2010) Outer-core compositional stratification from observed core wave speed profiles. Nature 468:807-810

Hirose K, Labrosse S, Hernlund J (2013) Composition and state of the core. Annu Rev Earth Planet Sci 41:657-691

Kaneshima S, Helffrich G (2013) Vp structure of the outermost core derived from analyzing large scale array data of SmKS waves. Geophys J Int 93:1537-1555

Kolafa J, Nezbeda I (1994) The Lennard-Jones fluid: an accurate analytic and theoretically-based equation of state. Fluid Ph Equil 100:1-34

Labrosse S, Poirier J-P, Mouël J-L (2001) The age of the inner core. Earth Planet Sci Lett 190:111-123

Mansoori GA, Carnahan NF, Starling KE, Leland TW (1971) Equilibrium thermodynamic properties of the mixture of hard spheres. J Chem Phys 54:1523-1525

Ohtaki T, Kaneshima S, Kanjo K (2012) Seismic structure near the inner core boundary in the south polar region. J Geophys Res 117:10-10292011008717

Protopapas P, Anderson HC, Parlee NAD (1973) Theory of transport in liquid metals. I. Calculation of self-diffusion coefficients. J Chem Phys 59:15-25

Rosenfeld Y (1999) Sound velocity in liquid metals and the hard-sphere model. J Phys Condens Matter 11:71-74

Shimoji M (1977) Liquid metals. Academic Press, London, p 391.

Smith DE, Zuber MT, Phillips RJ, Solomon SC, Steven A, Hauck I, Lemoine FG, Mazarico E, Neumann GA, Peale SJ, Margot J-L, Johnson CL, Torrence MH, Perry ME, Rowlands DD, Goossens S, Head JW, Taylor AH (2012) Gravity field and internal structure of mercury from MESSENGER. Science 336:214-217

Stacey FD, Davis PM (2008) Physics of the Earth. Cambridge Univ. Press, Cambridge

Stevenson DJ (1980) Application of liquid state physics to the Earth's core. Phys Earth Planet Inter 22:42-52

Tanaka S (2007) Possibility of a low P-wave velocity layer in the outermost core from global SmKS waveforms. Earth Planet Sci Lett 259:486-499

Yokoyama, I (2001a) Velocity of sound in liquid $3 d$ transition metals near the melting point. Mater Trans 42:2021-2023

Yokoyama I (2001b) Temperature dependence of sound velocity and self-diffusion coefficient in liquid alkali metals: a hard-sphere description. Physica B 293:338-342

Zou Z, Koper KD, Cormier VF (2008) The structure of the base of the outer core inferred from seismic waves diffracted around the inner core. J Geophys Res 113:10-10292007005316

\section{Submit your manuscript to a SpringerOpen ${ }^{\odot}$ journal and benefit from:}

- Convenient online submission

- Rigorous peer review

- Immediate publication on acceptance

- Open access: articles freely available online

- High visibility within the field

- Retaining the copyright to your article

Submit your next manuscript at $>$ springeropen.com 